\title{
Synthesis and Biological Evaluation of Some New 4(3h)-Quinazolinone Derivatives as Non-Classical Antifolate
}

\author{
M.A. El-Hashash, A.M. El-Metwally", A.M.F. Eissa ${ }^{* * *}$ and \\ A.M.F. El-Gohary ${ }^{*}$ \\ Faculty of Science, Ain Shams University, "National \\ Organization for Drug Control and Research and ${ }^{* *}$ Faculty of \\ Science, Banha University, Egypt.
}

\begin{abstract}
LL living cell need tetrahydrofolate cofactor for the synthesis of purines, some amino acid and thymidine. Most bacteria and plant produce this folate cofactor by de novo biosynthesis. Compounds that interfere with this pathway, antifolate agents have found use as anticancer. Thus, the 2-propyl-4H-3,1-benzoxazin-4-one (2) was synthesized and allowed to interaction with Ammonium acetate or formamide afforded -2- propylquinazolin-4(3H)-one (3). Behaviour of quinazolinone towards carbon electrophiles namely, aromatic aldehyde, chloroacetylchloride, and ethylchloroacetate has been investigated and all the synthesized compounds were tested as anti-cancer in National Cancer Institute (NCI) in USA.
\end{abstract}

Keywords: Benzoxazin-4-one, Quinazolin-4(3H) one and Anthranil .

The synthesis of quinazolinone heterocycles has become the cornerstone for synthetic chemists and gained extensive importance in medicinal chemistry because of their diverse pharmacological activities including antimycobacterial ${ }^{(1,2)}$, antimalarial $^{(3)}$, antihypertensive ${ }^{(4)}$, antihistaminic ${ }^{(5-9)}$, local anesthetic ${ }^{(10)}$, antiparkinson $^{(11)}$, cardiotonic ${ }^{(12)}$, anticancer ${ }^{(13-16)}$, antiviral ${ }^{(17)}$ and thymidylate synthase inhibitory activities ${ }^{(18)}$. Several simple and condensed quinazolines are also known to exhibit potent CNS activities as analgesic ${ }^{(19,20)}$, anti-inflammatory ${ }^{(21,22)}$ and anticonvulsant activities ${ }^{(23,24)}$. Besides these, the quinazolinone skeleton is frequently encountered as building block for hundreds of naturally occurring alkaloids ${ }^{(25)}$ and hence the exploration of this skeleton as privileged new chemical entities (NCEs) in drug discovery research is beyond doubt of paramount importance for the synthesis chemist. One of the most important features in 4H-3,1-benzoxazinones chemistry is their use as key starting materials for further transformations. With the aim of the extending information on the reactivity of 2-propyl-4H-3, 1-benzoxazinone and also synthesizing from them new quinazolin-4-ones systems, potentially with biological activity and in continuation of our work on the behavior of 3-propylquinazolin-4-one towards carbon nucleophiles, other derivatives were obtained via the interaction of hydrazide derivative 9 with acetic anhydride, acetylacetone, aromatic aldehydes, 3nitrophthalic anhydride and ethyl acetoacetate.

*Corresponding author: E-mail:dr.amiraalmetwaly@ hotmail.com 


\section{Results and Discussion}

2-Propyl-4H-benzo[d][1,3]ozazin- 4-one (2) has been synthesized from the interaction of butyroyl chloride with anthranilic acid in pyridine and yielded, 2butyramidobenzoic acid (1), ring closure of the acid 1 by using acetic anhydride afforded the desired benzoxazinone derivative 2 the structure of acid (1) was proved from its microanalytical data and its IR spectra $\left(\mathrm{cm}^{-1}\right)$ which showed strong absorption bands at 1672, 1693, 3286 and 3422 attributable to $v_{\max }$ of two carbonyl groups, $\mathrm{NH}$ and $\mathrm{OH}$ of acid. The structure of compound 2 was inferred from its IR spectrum which exhibits strong absorption bands at 1614, $1764\left(\mathrm{~cm}^{-1}\right)$ due to $v_{\max }$.of $\mathrm{C}==\mathrm{N}$ and $\mathrm{C}==\mathrm{O}$ of benzoxazinone and lack of any band for $\mathrm{NH}$ and / or $\mathrm{OH}$.

The key starting material 2-propylquinazolin-4(3H)-one (3)was synthesized by two methods; the first by heating compound 2 with ammonium acetate in an oil bath and the second method was carried by refluxing of compound 2 in formamide. The structure of compound 3 was inferred from its IR spectrum which exhibits strong absorption bands at 1614,1670 and $3200,\left(\mathrm{~cm}^{-1}\right)$ due to $v_{\max }$. of $\mathrm{C}==\mathrm{N}, \mathrm{C}==\mathrm{O}$ and $\mathrm{NH}$ and of quinazolinone and lack of any band for $\mathrm{C}=\mathrm{O}$ of benzoxazinone.

2-Propylquinazolin-4 $(3 \mathrm{H})$ - one (3) reacts with ethylchlorocaetate in the presence of anhydrous $\mathrm{K}_{2} \mathrm{CO}_{3}$ in boiling acetone afforded ethyl 2-(4-oxo-2propylquinazolin-3(4H)-yl)acetate (4). Treatment of ester 4 with secondary amine namely (piperidine and/or morpholine) gave 3-(2-piperidino or morpholino-2-oxoethyl)-2-propylquinazolin-4(3H)-one (5a-b). On the other hand, when compound 3 was allowed to react with chloroacetylchloride yielded 3-(2-chloroacetyl)-2-propylquinazolin-4 $(3 \mathrm{H})$-one $(6)$ which on treatment with hydrazine hydrate afforded the bis compound 7 . Interaction of the quinazolinone 3 with aldehydes in glacial acetic acid affording (E) 2-(1-aryl)but-1-en-2yl)quinazolin-4(3H)-one (8a-d). On the other hand, the ester 4 was treated with hydrazine hydrate afforded the corresponding hydrazide derivatives (9). Compound (9) was used for the synthesis of diverse heterocyclic compound, thus when compound (9) was allowed to react with boiling acetic anhydride yielding 3 - ( ( 5- methyl- 1, 3, 4 - oxadiazol-2 -yl ) methyl)- 2-propylquinazolin-4(3H)one (10). Acetylacetone reacts with hydrazide derivative 9 to give $3-(2-(3,5-$ dimethyl - 1H - pyrazol -1-yl) -2-oxoethyl) -2-propylquinazolin-4(3H)-one (11), also when compound 9 was allowed to react with ethylacetoacetate in boiling ethanol yielded 3-(5-(2-oxopropyl)-1,3,4-oxadiazol-2-yl)-2-propylquinazolin$4(3 \mathrm{H})$-one (12). When compound 9 was stirred with aromatic aldehyde namely,pipronal, p-hydroxybenzaldehyde and p-nitrobenzaldehyde at $70{ }^{\circ} \mathrm{C}$ in ethanol affording the corresponding arylidene aminoquinazolinone (13a-c). $\mathrm{N}-(4-$ nitro-1,3-dioxoisoindolin-2-yl)-2-(4-oxo-2- propylquinazolin $-3 \quad(4 \mathrm{H}) \quad-y l)$ acetamide (14) was obtaind by refluxing compound (9) with 3-nitrophthalic anhydride in ethanol.

Egypt. J. Chem. 53, No.6 (2010) 


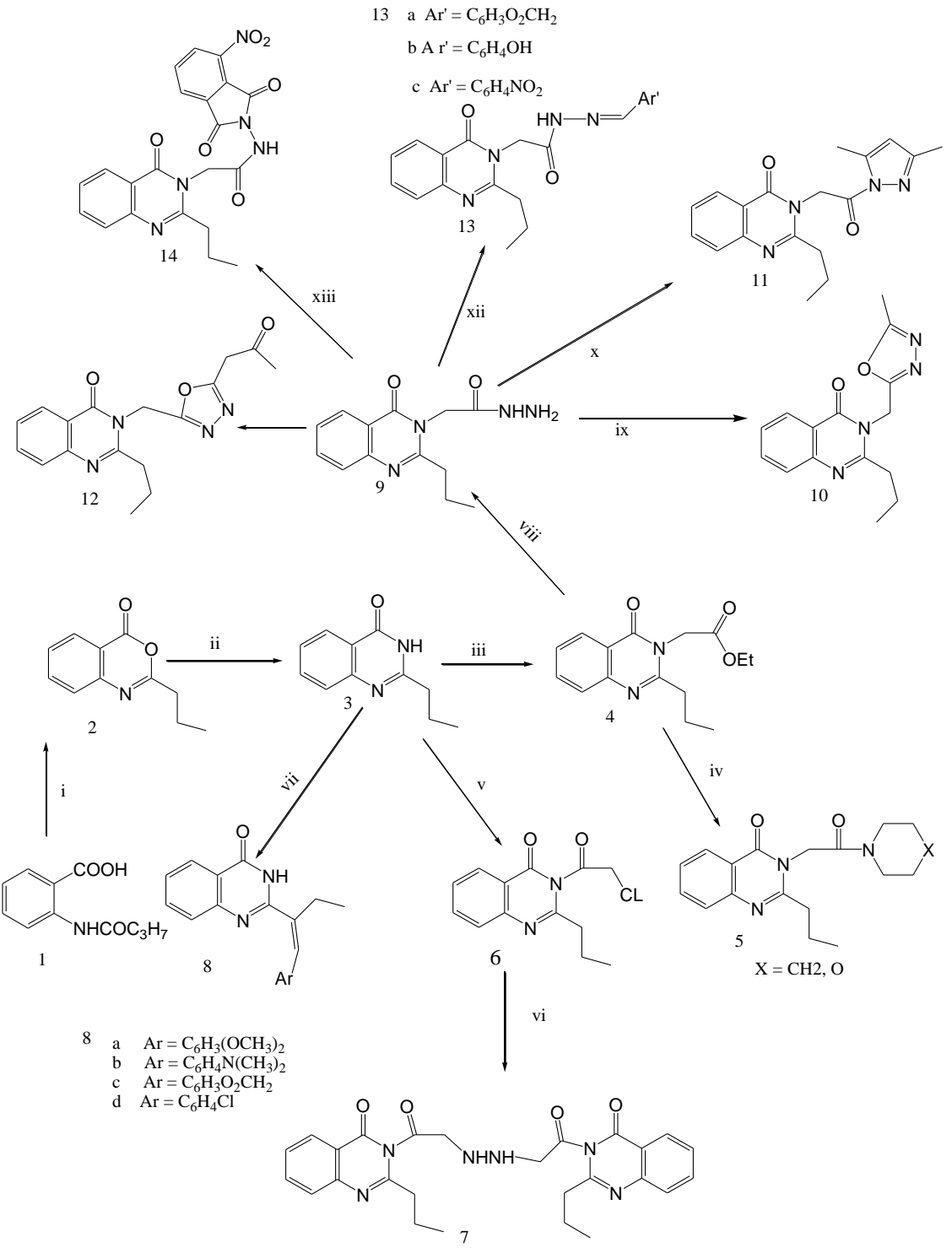

Scheme 1. Reagent and condition: (i) $\mathrm{Ac}_{2} \mathrm{O}, 140{ }^{\circ} \mathrm{C}$, (ii) $\mathrm{CH}_{3} \mathrm{COONH}_{4}, 150{ }^{\circ} \mathrm{C}$, (iii) $\mathrm{ClCH} 2 \mathrm{COOEt}$, anhydrous $\mathrm{K}_{2} \mathrm{CO}_{3}$, dry acetone, $56{ }^{\circ} \mathrm{C}$, (iv) piperidine/ morpholine, $\mathrm{C}_{2} \mathrm{H}_{5} \mathrm{OH}, 78{ }^{\circ} \mathrm{C}$, (v) $\mathrm{ClCH} 2 \mathrm{COCl}$, pyridine $115{ }^{\circ} \mathrm{C}$, (vi) $\mathrm{NH}_{2} \mathrm{NH}_{2}$, $\mathrm{C}_{2} \mathrm{H}_{5} \mathrm{OH}, 78{ }^{\circ} \mathrm{C}$, (vii) $\mathrm{ArCHO}, \mathrm{CH}_{3} \mathrm{COOH} / \mathrm{CH}_{3} \mathrm{COONa}$ (viii) $\mathrm{NH}_{2} \mathrm{NH}_{2}$, $\mathrm{C}_{2} \mathrm{H}_{5} \mathrm{OH}, 78{ }^{\circ} \mathrm{C}$, (ix) $\mathrm{Ac}_{2} \mathrm{O}, 140{ }^{\circ} \mathrm{C}$, (x) $\mathrm{Ac}_{2} \mathrm{CH}_{2}, \mathrm{C}_{2} \mathrm{H}_{5} \mathrm{OH}, 78{ }^{\circ} \mathrm{C}$, (xi) EAA, $\mathrm{C}_{2} \mathrm{H}_{5} \mathrm{OH}, 78{ }^{\circ} \mathrm{C}$, (xii) $\mathrm{Ar}{ }^{\prime} \mathrm{CHO}, \mathrm{C}_{2} \mathrm{H}_{5} \mathrm{OH}, 65^{\circ} \mathrm{C}$, (xiii) 3-nitrophthalic anhydride, $\mathrm{C}_{2} \mathrm{H}_{5} \mathrm{OH}, 78^{\circ} \mathrm{C}$.

Egypt. J. Chem. 53, No.6 (2010) 
TABLE 1. Characterization and physical data of synthesized compounds.

\begin{tabular}{|c|c|c|c|c|c|}
\hline \multirow{2}{*}{$\begin{array}{l}\text { Comp } \\
\text { No. }\end{array}$} & \multirow{2}{*}{$\begin{array}{l}\text { M.P. } \\
\text { C }^{\mathbf{0}}\end{array}$} & \multirow{2}{*}{ Solvent } & \multirow{2}{*}{$\begin{array}{c}\text { Formula } \\
\text { M. wt. }\end{array}$} & \multicolumn{2}{|c|}{ Analysis \%calc/found } \\
\hline & & & & $\mathbf{C}$ & $\mathbf{H}$ \\
\hline 1 & 125 & benzene & $\begin{array}{c}\mathrm{C}_{11} \mathrm{H}_{13} \mathrm{NO}_{3} \\
207\end{array}$ & $\begin{array}{l}63.76 \\
63.62\end{array}$ & $\begin{array}{l}6.28 \\
5.05\end{array}$ \\
\hline 2 & 59 & $\begin{array}{l}\text { Petroleum } \\
\text { ether40-60 }\end{array}$ & $\begin{array}{c}\mathrm{C}_{11} \mathrm{H}_{11} \mathrm{NO}_{2} \\
189\end{array}$ & $\begin{array}{l}69.84 \\
69.25\end{array}$ & $\begin{array}{l}5.82 \\
5.12\end{array}$ \\
\hline 3 & 195 & $\mathrm{DMF}$ & $\begin{array}{c}\mathrm{C}_{11} \mathrm{H}_{12} \mathrm{~N}_{2} \mathrm{O} \\
188\end{array}$ & $\begin{array}{l}70.21 \\
69.95\end{array}$ & $\begin{array}{l}6.38 \\
6.52\end{array}$ \\
\hline 4 & 105 & $\begin{array}{l}\text { Petroleum } \\
\text { ether60-80 }\end{array}$ & $\begin{array}{c}\mathrm{C}_{15} \mathrm{H}_{18} \mathrm{~N}_{2} \mathrm{O}_{3} \\
274 \\
\end{array}$ & $\begin{array}{l}65.69 \\
65.60 \\
\end{array}$ & $\begin{array}{l}6.56 \\
6.49 \\
\end{array}$ \\
\hline $5 \mathrm{a}$ & 119 & Methanol & $\begin{array}{c}\mathrm{C}_{18} \mathrm{H}_{23} \mathrm{~N}_{3} \mathrm{O}_{2} \\
313 \\
\end{array}$ & $\begin{array}{l}69.00 \\
69.11 \\
\end{array}$ & $\begin{array}{l}7.34 \\
7.23\end{array}$ \\
\hline $5 b$ & 116 & Ethanol & $\begin{array}{c}\mathrm{C}_{17} \mathrm{H}_{21} \mathrm{~N}_{3} \mathrm{O}_{3} \\
315 \\
\end{array}$ & $\begin{array}{l}64.76 \\
64.72 \\
\end{array}$ & $\begin{array}{l}3.34 \\
3.45 \\
\end{array}$ \\
\hline 6 & 285 & Ethanol & $\begin{array}{c}\mathrm{C}_{13} \mathrm{H}_{13} \mathrm{~N}_{2} \mathrm{O}_{2} \mathrm{Cl} \\
264.5\end{array}$ & $\begin{array}{l}58.97 \\
58.52 \\
\end{array}$ & $\begin{array}{l}4.9 \\
4.86 \\
\end{array}$ \\
\hline 7 & 202 & Ethanol & $\begin{array}{c}\mathrm{C}_{26} \mathrm{H}_{28} \mathrm{~N}_{6} \mathrm{O}_{4} \\
488\end{array}$ & $\begin{array}{l}63.93 \\
63.87 \\
\end{array}$ & $\begin{array}{l}5.73 \\
5.72 \\
\end{array}$ \\
\hline $8 a$ & 160 & benzene & $\begin{array}{c}\mathrm{C}_{20} \mathrm{H}_{20} \mathrm{~N}_{2} \mathrm{O}_{3} \\
336\end{array}$ & $\begin{array}{l}71.42 \\
71.31 \\
\end{array}$ & $\begin{array}{l}5.95 \\
5.89 \\
\end{array}$ \\
\hline $8 b$ & 150 & Methanol & $\begin{array}{c}\mathrm{C}_{20} \mathrm{H}_{21} \mathrm{~N}_{3} \mathrm{O} \\
319\end{array}$ & $\begin{array}{l}75.23 \\
75.36 \\
\end{array}$ & $\begin{array}{l}6.58 \\
6.56 \\
\end{array}$ \\
\hline $8 c$ & 145 & Methanol & $\begin{array}{c}\mathrm{C}_{19} \mathrm{H}_{16} \mathrm{~N}_{2} \mathrm{O}_{3} \\
320 \\
\end{array}$ & $\begin{array}{l}71.25 \\
71.29 \\
\end{array}$ & $\begin{array}{l}5.00 \\
4.90 \\
\end{array}$ \\
\hline $8 \mathrm{~d}$ & 151 & Methanol & $\begin{array}{c}\mathrm{C}_{18} \mathrm{H}_{15} \mathrm{~N}_{2} \mathrm{OCl} \\
310.5\end{array}$ & $\begin{array}{l}69.56 \\
69.66\end{array}$ & $\begin{array}{l}4.83 \\
4.79\end{array}$ \\
\hline 9 & 165 & Ethanol & $\begin{array}{c}\mathrm{C}_{13} \mathrm{H}_{16} \mathrm{~N}_{4} \mathrm{O}_{2} \\
260\end{array}$ & $\begin{array}{l}60.00 \\
59.94\end{array}$ & $\begin{array}{l}6.15 \\
6.19\end{array}$ \\
\hline 10 & 145 & benzene & $\begin{array}{c}\mathrm{C}_{15} \mathrm{H}_{16} \mathrm{~N}_{4} \mathrm{O}_{2} \\
284\end{array}$ & $\begin{array}{l}63.38 \\
63.64\end{array}$ & $\begin{array}{l}5.63 \\
5.71\end{array}$ \\
\hline 11 & 195 & Ethanol & $\begin{array}{c}\mathrm{C}_{18} \mathrm{H}_{20} \mathrm{~N}_{4} \mathrm{O}_{2} \\
356\end{array}$ & $\begin{array}{l}60.67 \\
60 . .49\end{array}$ & $\begin{array}{l}5.61 \\
5.54 \\
\end{array}$ \\
\hline 12 & 265 & Ethanol & $\begin{array}{c}\mathrm{C}_{17} \mathrm{H}_{18} \mathrm{~N}_{4} \mathrm{O}_{3} \\
326\end{array}$ & $\begin{array}{l}62.57 \\
62.57\end{array}$ & $\begin{array}{l}5.52 \\
5.51\end{array}$ \\
\hline $13 \mathrm{a}$ & 245 & Ethanol & $\begin{array}{c}\mathrm{C}_{21} \mathrm{H}_{20} \mathrm{~N}_{4} \mathrm{O}_{4} \\
392\end{array}$ & $\begin{array}{l}64.28 \\
64.31\end{array}$ & $\begin{array}{l}5.10 \\
5.21\end{array}$ \\
\hline $13 b$ & 174 & Ethanol & $\begin{array}{c}\mathrm{C}_{20} \mathrm{H}_{20} \mathrm{~N}_{4} \mathrm{O}_{3} \\
364\end{array}$ & $\begin{array}{l}65.93 \\
65.88\end{array}$ & $\begin{array}{l}5.49 \\
5.40\end{array}$ \\
\hline $13 \mathrm{c}$ & 260 & Ethanol & $\begin{array}{c}\mathrm{C}_{20} \mathrm{H}_{19} \mathrm{~N}_{5} \mathrm{O}_{4} \\
393 \\
\end{array}$ & $\begin{array}{l}61.06 \\
61.10 \\
\end{array}$ & $\begin{array}{l}4.83 \\
4.81 \\
\end{array}$ \\
\hline 14 & 250 & Methanol & $\begin{array}{c}\mathrm{C}_{21} \mathrm{H}_{17} \mathrm{~N}_{5} \mathrm{O}_{6} \\
435\end{array}$ & $\begin{array}{l}57.93 \\
57.82\end{array}$ & $\begin{array}{l}3.90 \\
3.82\end{array}$ \\
\hline
\end{tabular}

Egypt. J. Chem. 53, No.6 (2010) 


\section{Biological Part}

The 1960s saw the emergence of unique class of DHFR inhibitors. These quinazoline and pyrimidine analogues of folic acid are called nonclassical or lipophilic because they lack the glutamate residue found in classical DHFR inhibitors like Methotrexate (MTX) ${ }^{(26)}$. All compounds were evaluated in the National Cancer Institute (NCI), USA, Invitro preclinical antitumor screening program and inhibited the growth of tumor cells in cultcure micromoles to submicromoles concentrations.

\section{Evaluation of cytotoxicity of Quinazolin-4-ones derivatives}

\section{In-vitro}

The Development Therapeutics Program (DTP), Division of Cancer Treatment and Diagnosis (DCTD), National Cancer Institute (NCI) USA has used an Invitro model consisting of 60 human tumor cell lines as the primary anti-cancer screen. An analysis of the data indicated that approximately $95 \%$ of the actives from the 60 -cell line screen could be identified using only three cell lines. For this reason, the DTP has begun using, as its primary anti-cancer assay, a 3-cell lines panel consisting of NCI-H 460(Lung), MCF7 (Breast), and SF-268 (CNS). The NCI protocol has been described previously briefly. Cell lines were inoculated onto a series of 96-well plates. Seeding densities varied depending upon growth characteristics. After a $24 \mathrm{hr}$ - drug - free incubation, test compounds were added routinely at five ten fold dilutions starting at maximum 10-4M. After incubation periods of $48 \mathrm{hr}$ or 6 days, cell growth or viability was assayed using the sulphorhodamine B procedure. From the results of antineoplastic evaluation of the tested compounds it is evident that compounds (8a-d) , namely compound (8a) showed cytotoxic effects and high selectivity against Malanoma (SK-MEL-2), Renal cancer (TK-10) and Ovarian cancer (IGROV1). Compound (8b) showed cytotoxic effects and high selectivity against Prostate cancer(PC-3) and Renal cancer (TK-10), compounds (8c) showed cytotoxic effects and high selectivity against Renal cancer (TK-10) and Colon cancer (HCC-2998) and Compound (8d) showed cytotoxic effects and high selectivity against Renal cancer (TK-10), Prostate cancer (PC-3) and CNS cancer (SF-295).Compound 10 showed cytotoxic effects and high selectivity against Breast cancer (MCF7) and Leukemia (SR), also compound 11 showed cytotoxic effects and high selectivity against Colon cancer (HT29) and CNS cancer (SF268). Evaluation of cytotoxicity of some synthesized compounds against human cancer cell line (In-vitro) is shown in Table 2. 
TABLE 2. Evaluation of cytotoxicity of some synthesized compound against human cancer cell line (In-vitro).

\begin{tabular}{|c|c|c|c|}
\hline Comp. No. & NSC & Cancer cell line activity againest & LogGI 50 \\
\hline \multirow{2}{*}{ 8a } & 746764 & Malanoma (SK-MEL-2), & 4.91 \\
& & Renal cancer (TK-10) and & -5.23 \\
Mean value & & Ovarian cancer (IGROV1) & -4.86 \\
\hline \multirow{2}{*}{$\mathbf{b}$} & 746765 & Prostate cancer (PC-3), & -4.86 \\
\hline \multirow{2}{*}{$\mathbf{8 c}$} & 746855 & Renal cancer (TK-10) & -5.88 \\
Mean value & & Renal cancer (TK-10), & -5.00 \\
\hline \multirow{2}{*}{ 8d } & 746856 & Colon cancer (HCC-2998) & -4.91 \\
& & Renal cancer (TK-10), & -4.96 \\
Mean value & & Prostate cancer (PC-3) & -4.73 \\
\hline \multirow{2}{*}{ 10 } & 746857 & CNS cancer (SF-295) & -4.68 \\
Mean value & & Breast cancer (MCF7) & -4.56 \\
\hline \multirow{2}{*}{ 11 } & 746858 & Leukemia (SR) & -4.55 \\
Mean value & & Colon cancer (HT29) & -4.54 \\
\hline
\end{tabular}

$\log \mathrm{GI}_{50}$ : growth inhibition effect of fifty percent.

\section{Experimental Part}

All melting points are uncorrected and determined by the open capillary method using Gallen Kamp melting point apparatus. Microanalyses were carried out by the Micro Analytical Unit at Cairo University. IR spectra (KBr disk) were recorded on FT/IR-300E Jasco spectrophotometer. HNMR spectra were recorded in $\mathrm{CDCl}_{3}$ or DMSO-d $\mathrm{d}_{6}$ solution on a Varian EM $390-90 \mathrm{MHz}$. Mass spectrometry were recorded on Shomadzu, GC - MS (QP - 1000EX.

\section{Butyramidobenzoic acid (1)}

A stirred solution of 2-aminobenzoic acid $13.7 \mathrm{~g}(0.1 \mathrm{~mol})$ in dry pyridine $(150 \mathrm{ml})$ was treated drop wise with $\mathrm{n}$-butyroyl chloride $10.5 \mathrm{~g}(0.11 \mathrm{~mol})$ during $10 \mathrm{~min}$ the mixture was stirred at room temperature $(3 \mathrm{hr})$ and poured into a mixture of ice and hydrochloric acid to give . 2-butyramidobenzoic acid IR ( $\mathrm{KBr})$ $\mathrm{cm}^{-1}: 3422(\mathrm{OH}), 3286(\mathrm{NH}), 3055(\mathrm{CH}$ aromatic $), 2957,2927,2869(\mathrm{CH}$ aliphatic), 1693 ( $\mathrm{c}=\mathrm{o}$, acid), 1672 ( $\mathrm{c}=\mathrm{o}$, amide).

\section{2-Propyl-4H- benzo[d][1,3]oxazin-4-one (2)}

A suspension of 2-butyramidobenzoic acid $2.07 \mathrm{~g}(0.01 \mathrm{~mol})$ in $50 \mathrm{ml}$ acetic anhydride was heated under reflux ( $3 \mathrm{hr}$ ) and then concentrated. The residue was crystallized from petroleum ether $40-60^{\circ} \mathrm{C}$, giving 2-propyl-4H- 
benzo[d][1,3]oxazin-4-one as a colorless crystals melting point $\left(59^{\circ} \mathrm{C}\right)$. IR $(\mathrm{KBr})$ $\mathrm{cm}^{-1}: 3055$ (CH aromatic), 2965, 2935, 2875 (CH aliphatic), 1764 (c=o), $1614(\mathrm{C}=\mathrm{N}), 1161(\mathrm{C}-\mathrm{O})$.

2-Propylquinazolin-4(3H)-one (3)

Method A

2-Propyl-4H- benzo[d][1,3]oxazin-4-one(2) (1 mmol) was heated with ammonium acetate $(4 \mathrm{mmol})$ in an oil bath at $150^{\circ} \mathrm{C}$ for $2 \mathrm{hr}$, the reaction mixture was cooled and poured in cold water. The precipitated solid was filtered off , washed with water and recrystallization from the proper solvent to give (3) $\mathrm{IR}(\mathrm{KBr}) \mathrm{cm}^{-1}: 3215(\mathrm{NH}), 3024(\mathrm{CH}$ aromatic), 2969, 2935, 2905, $2877(\mathrm{CH}$ aliphatic), $1675(\mathrm{C}=\mathrm{O}), 1597(\mathrm{C}=\mathrm{N}) ; \mathrm{MS}: \mathrm{m} / \mathrm{z} 189\left[\mathrm{M}^{+}\right], 187,172,158,144,130$; ${ }^{1} \mathrm{HNMR}\left(\mathrm{CHCl}_{3}\right): \delta 7.21-8.23\left(\mathrm{~m}, 4 \mathrm{H}\right.$, aromatic), 1.14(t, 3H, $\left.\underline{\mathrm{CH}}_{3}\right), 1.92$ (sextet,2H, $\underline{\mathrm{CH}}_{2} \mathrm{Me}$ ), $2.72\left(\mathrm{t}, 2 \mathrm{H}, \underline{\mathrm{CH}}_{2} \mathrm{Q}\right.$ ).

\section{Method B}

2-Propyl-4H- benzo[d][1,3]oxazin-4-one(2) $(1 \mathrm{mmol})$ in formamide $(20 \mathrm{ml})$ was refluxed for $3 \mathrm{hr}$, the reaction mixture was cooled and poured in cold water. The obtained solid was filtered off and recrystalized from the proper solvent IR(KBr) cm $\mathrm{cm}^{-1}: 3215(\mathrm{NH}), 3024$ (CH aromatic), 2969, 2935, 2905, $2877(\mathrm{CH}$ aliphatic), $1675(\mathrm{C}=\mathrm{O}), 1597(\mathrm{C}=\mathrm{N}) ; \mathrm{MS}: \mathrm{m} / \mathrm{z} 189\left[\mathrm{M}^{+}\right], 187,172,158,144,130$; ${ }^{1} \mathrm{HNMR}\left(\mathrm{CHCl}_{3}\right): \delta 7.21-8.23\left(\mathrm{~m}, 4 \mathrm{H}\right.$, aromatic), 1.14(t, 3H, $\left.\underline{\mathrm{CH}}_{3}\right), 1.92$ (sextet,2H, $\mathrm{CH}_{2} \mathrm{Me}$ ), $2.72\left(\mathrm{t}, 2 \mathrm{H}, \underline{\mathrm{CH}_{2}} \mathrm{Q}\right.$ ).

Ethyl 2-(4-oxo-2-propylquinazolin-3(4H)-yl) acetate (4)

A suspension of 2-propylquinazolin-4(3H)-one $(0.01 \mathrm{~mol})$ in $50 \mathrm{ml}$ dry acetone was stirred and potassium carbonate $(0.04 \mathrm{~mol})$ was added after the mixture was stirred at room temperature $20 \mathrm{~min}$, the ethylcloroacetate $(0.02 \mathrm{~mol})$ was added, and the mixture was refluxed $24 \mathrm{hr}$. The solvent was evaporated under reduced pressure, water was added to the residue and the produced solid was filtered off and recrystallized from petroleum ether $60-80{ }^{\circ} \mathrm{C}$ yield (4) m. p. $105^{\circ} \mathrm{C}$. IR(KBr) cm $\mathrm{cm}^{-1}: 3024(\mathrm{CH}$ aromatic $), 2969,2935,2905,2877(\mathrm{CH}$ aliphatic), $1735(\mathrm{C}=\mathrm{O}$ ester ),1675 ( $\mathrm{C}=\mathrm{O}), 1597(\mathrm{C}=\mathrm{N}), 1232$ (C-O ester); MS: $\mathrm{m} / \mathrm{z} 275\left[\mathrm{M}^{+}\right], 273,258,244,229,215,172,158,142 ;{ }^{1} \mathrm{HNMR}\left(\mathrm{CHCl}_{3}\right): \delta$ $7.21-8.23$ (m, 4H, aromatic), $4.92\left(\mathrm{~s}, 2 \mathrm{H}, \mathrm{QCH}_{2} \mathrm{CO}\right), 4.32\left(\mathrm{q}, 2 \mathrm{H}, \mathrm{OCH}_{2}\right) 1.33(\mathrm{t}$, $\left.3 \mathrm{H}, \mathrm{OCH}_{2} \mathrm{CH}_{3}\right)$ 1.14(t, 3H, $\mathrm{CH}_{3}$ ), 1.92 (sextet, $2 \mathrm{H}, \mathrm{CH}_{2} \mathrm{Me}$ ), $2.72\left(\mathrm{t}, 2 \mathrm{H}, \underline{\mathrm{CH}_{2}} \mathrm{Q}\right.$ ).

3-(2-Piperidino or morpholino-2-oxoethyl)-2-propylquinazolin-4(3H)-one (5a- b)

A mixture of $4(0.01 \mathrm{~mol})$ and of piperidine or morpholine $(10 \mathrm{ml})$ was refluxed for $8 \mathrm{hr}$. The produced mixture was cooled, evaporated under reduced pressure and water was added to the residue and the produced solid was filtered off and recrystallized from the proper solvent.

3-(2-Oxo-2-piperidin-1-yl)ethyl)-2-oxoethyl)-2-propylquinazolin-4(3H)-one (5a)

$\operatorname{IR}(\mathrm{KBr}) \mathrm{cm}^{-1}: 3040$ (CH aromatic), 2956, 2906, 2868, (CH aliphatic), 1700, $1675(2 \mathrm{C}=\mathrm{O}), 1610(\mathrm{C}=\mathrm{N}) ; \mathrm{MS}: \mathrm{m} / \mathrm{z} 313$,311, 296,281, 269, 244, 229, 215, 172, 
$158,171,142 ;{ }^{1} \mathrm{HNMR}\left(\mathrm{CHCl}_{3}\right): \delta 7.2-8.23(\mathrm{~m}, 4 \mathrm{H}$, aromatic), 3.22 (s, 4H, $\mathrm{N}\left(\mathrm{CH}_{2}\right)_{2}$ piperidine), 1.84 (pentet, $6 \mathrm{H}$, piperidine) $1.14\left(\mathrm{t}, 3 \mathrm{H}, \underline{\mathrm{CH}}_{3}\right), 1.92$ (sextet, $\left.2 \mathrm{H}, \mathrm{CH}_{2} \mathrm{Me}\right), 2.72\left(\mathrm{t}, 2 \mathrm{H}, \underline{\mathrm{CH}_{2}} \mathrm{Q}\right)$.

3-(2-Morpholino-2-oxoethyl)-2-propylquinazolin-4(3H)-one (5b)

$\mathrm{IR}(\mathrm{KBr}) \mathrm{cm}^{-1}: 3040$ (CH aromatic), 2956, 2906, 2868, (CH aliphatic), 1695, $1672(2 \mathrm{C}=\mathrm{O}), 1605(\mathrm{C}=\mathrm{N}) ; \mathrm{MS}: \mathrm{m} / \mathrm{z} 316 \quad\left[\mathrm{M}^{+}\right], 314$ 299, 285, 271, 257, 171, 142; ${ }^{1} \mathrm{HNMR}\left(\mathrm{DMSO}_{6}\right): \delta 8.14(\mathrm{~s}, 1 \mathrm{H}, \mathrm{NH}), 7.22-7.73(\mathrm{~m}, 4 \mathrm{H}$, aromatic), $3.81\left(\mathrm{t}, 4 \mathrm{H}, \mathrm{O}\left(\mathrm{CH}_{2}\right)_{2}\right.$ morphiline $), 3.22\left(\mathrm{t}, 4 \mathrm{H}, \mathrm{N}\left(\mathrm{CH}_{2}\right)_{2}\right.$ morphiline), 1.14(t, $3 \mathrm{H}$, $\underline{\mathrm{CH}}_{3}$ ), 1.92 (sextet,2H, $\underline{\mathrm{CH}}_{2} \mathrm{Me}$ ), $2.72\left(\mathrm{t}, 2 \mathrm{H}, \underline{\mathrm{CH}}_{2} \mathrm{Q}\right.$ ).

\section{3-(2-Chloroacetyl)-2-propylquinazolin-4(3H)-one (6)}

Compound $3(0.01 \mathrm{~mol})$ was dissolved in pyridine $30 \mathrm{ml}$ and the mixture was stirred, chloroacetyl chloride was added dropwise at room temperature, and the mixture was continued in stirring for $3 \mathrm{hr}$. Then the mixture was poured on ice and $\mathrm{HCl}$, the precipitate was collected by filtration and recrystallization from proper solvent. M.p.285. IR(KBr) cm $\mathrm{cm}^{-1}$ : 3040 (CH aromatic), 2956, 2906, 2868, (CH aliphatic), 1710, $1668(2 \mathrm{C}=\mathrm{O}), 1605(\mathrm{C}=\mathrm{N})$; MS: m/z 264.5 , 230, 215 201, 187, 153, $142 ;{ }^{1} \mathrm{HNMR}\left(\mathrm{DMSO}_{6}\right): \delta 7.22-7.73(\mathrm{~m}, 4 \mathrm{H}$, aromatic), $6.21(\mathrm{~s}$, $2 \mathrm{H}, \mathrm{COCH}_{2} \mathrm{Cl}$ ), 1.14(t, 3H, $\left.\underline{\mathrm{CH}}_{3}\right), 1.92$ (sextet, $\left.2 \mathrm{H}, \underline{\mathrm{CH}}_{2} \mathrm{Me}\right), 2.72\left(\mathrm{t}, 2 \mathrm{H}, \underline{\mathrm{CH}}_{2} \mathrm{Q}\right.$ ).

N,N'di(2-(4-oxo-2-propylquinazolin-3(4H)-yl)aceto)hydrazide(7)

A solution of compound $6(0.01 \mathrm{~mol})$ and hydrazine hydrate $(0.01 \mathrm{~mol})$ in ethanol $50 \mathrm{ml}$ was heated under reflux for $3 \mathrm{hr}$. The reaction mixture was allowed to cool, filtered off and recrystallized from proper solvent to give (7) m.p. $202^{\circ} \mathrm{C}$. $\mathrm{IR}(\mathrm{KBr}) \mathrm{cm}^{-1}$ : 3271, $3285(2 \mathrm{NH}), 3040$ (CH aromatic), 2956, 2906, 2868, (CH aliphatic), 1685-1665 (4C=O), $1605(\mathrm{C}=\mathrm{N}) ; \mathrm{MS}: \mathrm{m} / \mathrm{z} 489 \quad\left[\mathrm{M}^{+}\right], 485,470,456$, $335,299260,151,71$.

\section{(E)-2-(1-aryl)but-1-en-2-yl) quinazolin-4(3H)-one (8a-d)}

A solution of compound $3(1.5 \mathrm{mmol})$, appropriate aldehyde $(2 \mathrm{mmol})$ and sodium acetate $(0.2 \mathrm{~g})$ were dissolved in a mixture of $5 \mathrm{ml}$ of glacial acetic acid and $1 \mathrm{ml}$ of acetic anhydride and the mixture was heated at reflux overnight, the acetic acid mixture solvent was removed under reduce pressure and the residues were partitioned between aqueous sodium bicarbonate and chloroform. The organic phase was extracted and excess solvent was evaporated to give compounds (8a-d) which were recrystallized from proper solvent.

(E)2-(1-(3,4-dimethoxyphenyl)but-1-en-2-yl)quinazolin-4(3H)-one (8a)

$\mathrm{IR}(\mathrm{KBr}) \quad \mathrm{cm}^{-1}: 2200(\mathrm{NH}), 3076(\mathrm{CH}$ aromatic), 2962, 2919, $2870(\mathrm{CH}$ aliphatic),1670(c=o), 1607(C=N); MS: m/z $337\left[\mathrm{M}^{+}\right], 335,320,306,291,277,262$, 249, 158, 71; ${ }^{1} \mathrm{HNMR}\left(\mathrm{DMSO}_{\mathrm{d}}\right.$ ) $: \delta 12.12$ (s, H, NH), $7.71-8.12$ (m, 7H, aromatic), $5.69(\mathrm{~s}, 1 \mathrm{H}, \mathrm{C}=\underline{\mathrm{CH}}) 3.41\left(\mathrm{~s}, 6 \mathrm{H}, 2 \mathrm{OCH}_{3}\right), 1.22\left(\mathrm{t}, 3 \mathrm{H}, \underline{\mathrm{CH}_{3}}\right), 2.31\left(\mathrm{q}, 2 \mathrm{H}, \underline{\mathrm{CH}_{2}} \mathbf{M e}\right)$.

(E)-2-(1-(4-(dimethylamino) phenyl)but-1-en-2-yl)quinazolin-4(3H)-one (8b)

$\mathrm{IR}(\mathrm{KBr}) \mathrm{cm}^{-1}: 3210(\mathrm{NH}), 3063(\mathrm{CH}$ aromatic), 2975, 2925, $2875(\mathrm{CH}$ aliphatic),1671(C=O), $1606(\mathrm{C}=\mathrm{N}) ; \mathrm{MS}: \mathrm{m} / \mathrm{z} 319,318,303,278,263,247,158$; Egypt. J. Chem. 53, No.6 (2010) 
${ }^{1} \mathrm{HNMR}\left(\mathrm{DMSO}-\mathrm{d}_{6}\right): \delta 12.12(\mathrm{~s}, \mathrm{H}, \mathrm{NH}), 7.73-8.32(\mathrm{~m}, 8 \mathrm{H}$, aromatic), $5.69(\mathrm{~s}$, $1 \mathrm{H}, \mathrm{C}=\underline{\mathrm{CH}}), 3.34\left(\mathrm{~s}, 6 \mathrm{H}, \mathrm{N}\left(\underline{\mathrm{CH}}_{3}\right)_{2}\right), 1.32\left(\mathrm{t}, 3 \mathrm{H}, \underline{\mathrm{CH}_{3}}\right), 2.31\left(\mathrm{q}, 2 \mathrm{H}, \underline{\mathrm{CH}_{2}} \mathrm{Me}\right)$.

(E)-2-(1-(benzo[d][1,3]dioxol-5-yl)but-1-en-2-yl)quinazolin-4(3H)-one (8c)

$\mathrm{IR}(\mathrm{KBr}) \mathrm{cm}^{-1}: 3210(\mathrm{NH}), 3076(\mathrm{CH}$ aromatic), 2962, 2919, $2870(\mathrm{CH})$ aliphatic),1673(C=O), 1601(C=N), 1255 (C-O-C); MS: m/z $320,319,304,377$, 262,232, 216, 128; ${ }^{1} \mathrm{HNMR}$ (DMSO-d $\left.\mathrm{d}_{6}\right): \delta 12.12(\mathrm{~s}, \mathrm{H}, \mathrm{NH}), 7.71-8.12(\mathrm{~m}, 7 \mathrm{H}$, aromatic), $6.11(\mathrm{~s}, 1 \mathrm{H}, \mathrm{C}=\underline{\mathrm{CH}}), 4.21\left(\mathrm{~s}, 2 \mathrm{H}, \mathrm{O}-\mathrm{CH}_{2}-\mathrm{O}\right), 1.22\left(\mathrm{t}, 3 \mathrm{H}, \underline{\mathrm{CH}_{3}}\right), 2.31$ $\left(\mathrm{q}, 2 \mathrm{H}, \mathrm{CH}_{2} \mathrm{Me}\right)$.

(E)-2-(1-(4-chlorophenyl) but-1-en-2-yl)quinazolin-4(3H)-one (8d)

$\operatorname{IR}(\mathrm{KBr}) \mathrm{cm}^{-1}: 3200(\mathrm{NH}), 3066(\mathrm{CH}$ aromatic), 2976, 2925, $2888(\mathrm{CH})$ aliphatic), $1672(\mathrm{C}=\mathrm{O}), 1607(\mathrm{C}=\mathrm{N}) ;{ }^{1} \mathrm{HNMR}\left(\mathrm{DMSO}^{\mathrm{d}}{ }_{6}\right): \delta 12.12(\mathrm{~s}, \mathrm{H}, \mathrm{NH})$, $7.73-8.32\left(\mathrm{~m}, 8 \mathrm{H}\right.$, aromatic), $6.11(\mathrm{~s}, 1 \mathrm{H}, \mathrm{C}=\underline{\mathrm{CH}}), 1.33\left(\mathrm{t}, 3 \mathrm{H}, \underline{\mathrm{CH}_{3}}\right), 2.41$ $\left(\mathrm{q}, 2 \mathrm{H}, \mathrm{CH}_{2} \mathrm{Me}\right)$.

2-(4-Oxo-2-propylquinazolin-3(4H)-yl) acetohydrazide (9)

A solution of ester $4(0.01 \mathrm{~mol})$ and hydrazine hydrate $(0.01 \mathrm{~mol})$ in ethanol $(50 \mathrm{ml})$ was refluxed for $3 \mathrm{hr}$, the reaction mixture was concentrated and then allowed to cool. The obtained solid was filtered off and recrystallization from ethanol yield compound (9) m.p. $165^{\circ} \mathrm{C}$. IR $(\mathrm{KBr}) \mathrm{cm}^{-1}: 3312,3271(\mathrm{NH} 2), 3171$ (NH), 3055 (CH aromatic), 2962, 2931, 2870, (CH aliphatic), 1685 (C=O), 1675 (C=O Quinazolinone), 1597(C=N); MS: m/z 260, 259, 244, 230, 216, 185, 157, 143,144; 1HNMR (DMSO-d6): $\delta 10.5\left(\mathrm{~s}, \mathrm{H}, \mathrm{NH}_{2}\right.$ ), $7.22-7.71(\mathrm{~m}, 4 \mathrm{H}$, aromatic), $4.51(\mathrm{~s}, \mathrm{H}, \mathrm{NH}), 2.83\left(\mathrm{t}, 2 \mathrm{H}, \mathrm{CH}_{2} \mathrm{Q}\right) 1.52\left(\operatorname{sextet}, 2 \mathrm{H}, \mathrm{CH}_{2} \mathrm{Me}\right),, 1.11(\mathrm{t}$, $\left.3 \mathrm{H}, \mathrm{CH}_{3}\right)$.

3-((5-Methyl-1,3,4-oxadiazol-2-yl)methyl)-2-propylquinazolin-4(3H)-one (10)

A solution of compound $9(0.01 \mathrm{~mol})$ and acetic anhydride $30 \mathrm{ml}$ was refluxed for $3 \mathrm{hr}$, the reaction mixture was allowed to cool and the obtained solid was filtered off and recrystallized from proper solvent to yield compound 10 m.p. $145^{\circ} \mathrm{C} \mathrm{IR}(\mathrm{KBr}) \mathrm{cm}^{-1}: 3055$ (CH aromatic), 2962, 2931, 2870, (CH aliphatic), 1675 (C=O Quinazolinone), 1597, $1605(\mathrm{C}=\mathrm{N}) ; \mathrm{MS}: \mathrm{m} / \mathrm{z} 284,286,271,257$, 233, 201, 185, 157, 143,144. ; 1HNMR (DMSO-d6): $\delta$ 7.22-7.71 (m, 4H, aromatic), 2.83 (t,2H, $\mathrm{CH}_{2} \mathrm{Q} 1.52$ (sextet, $2 \mathrm{H}, \mathrm{CH}_{2} \mathrm{Me}$ ), , 1.11(t, $3 \mathrm{H}, \mathrm{CH}_{3}$ ).

3-(2-(3,5-Dimethyl-1H-pyrazol-1-yl)-2-oxoethyl)-2-propylquinazolin-4(3H)-one (11)

A solution of compound $9(0.01 \mathrm{~mol})$ and acetylacetone $(0.01 \mathrm{~mol})$ in ethanol $50 \mathrm{ml}$ was heated under reflux for $3 \mathrm{hr}$. The reaction mixture was allowed to cool, filtered off and recrystallized from proper solvent to give $11 \mathrm{~m} . \mathrm{p} .195$. IR (KBr) $\mathrm{cm}^{-1}: 3055$ (CH aromatic), 2962, 2931, 2870, (CH aliphatic), 1675 (C=O Quinazolinone), 1597, 1605 (C=N); MS: m/z 357 [M+], 324, 244, 309, 285, 271, 157, 143,144. ; 1HNMR (DMSO-d6) : $\delta$, $7.22-7.71$ (m, 4H, aromatic), 6.25 (s, $\left.2 \mathrm{H}, \underline{\mathrm{CH}_{2}} \mathrm{CO}\right), 4.51(\mathrm{~s}, \mathrm{H}, \mathrm{C}=\underline{\mathrm{CH}}) 2.94\left(\mathrm{~s}, 6 \mathrm{H}, \underline{\mathrm{CH}}_{3}\right.$ pyrazol), $2.83\left(\mathrm{t}, 2 \mathrm{H}, \underline{\mathrm{CH}}_{2} \mathrm{Q}\right) 1.52$ (sextet,2 $\left.\mathrm{H}, \underline{\mathrm{CH}}_{2} \mathrm{Me}\right), 1.11\left(\mathrm{t}, 3 \mathrm{H}, \underline{\mathrm{CH}}_{3}\right)$. 
3-((5-(2-Oxopropyl)-1,3,4-oxadiazol-2-yl)methyl)-2-propylquinazolin-4(3H)-one(12)

A solution of compound $9(0.01 \mathrm{~mol})$ and ethylacetoacetate $(0.01 \mathrm{~mol})$ in ethanol $50 \mathrm{ml}$ was heated under reflux for $3 \mathrm{hr}$. The reaction mixture was allowed to cool, filtered off and recrystallized from proper solvent to give (12) m.p. 265. $\mathrm{IR}(\mathrm{KBr}) \mathrm{cm}^{-1}: 3055$ (CH aromatic), 2962, 2931, 2870, (CH aliphatic), 1682 $\left(\mathrm{CH}_{2} \mathrm{C}=\mathrm{O}\right), 1700(\mathrm{C}=\mathrm{O}$ pyrazol $), 1675(\mathrm{C}=\mathrm{O}$ Quinazolinone $), 1597,1605(\mathrm{C}=\mathrm{N})$; MS: $\mathrm{m} / \mathrm{z} 327[\mathrm{M}+], 325,243,308,284,270,157,143,144,99 . ; 1 \mathrm{HNMR}$ (DMSO-d6) : $\delta, 7.22-7.71\left(\mathrm{~m}, 4 \mathrm{H}\right.$, aromatic), $6.25\left(\mathrm{~s}, 2 \mathrm{H}, \mathrm{CH}_{2} \mathrm{CO}\right), 5.41(\mathrm{~s}, \mathrm{H}$, CHCOpyrazol), 2.94 (s,3H, $\mathrm{CH}_{3}$ pyrazol), 2.83 (t,2H, $\left.\mathrm{CH}_{2} \mathrm{Q}\right) 1.52$ (sextet, $2 \mathrm{H}_{2} \mathrm{CH}_{2}$ $\mathrm{Me}), 1.11\left(\mathrm{t}, 3 \mathrm{H}, \mathrm{CH}_{3}\right)$.

(E)-N'-aryl-2-(4-oxo-2-propylquinazolin-3(4H)-yl)acetohydrazide (13 a-c)

A solution of hydrazide $9(0.01 \mathrm{~mol})$ and aldehyde namely, pipronal, phydroxybenzaldehyde and p-nitrobenzaldehyde $(0.01 \mathrm{~mol})$ in ethanol was stirred $3 \mathrm{hr}$ at $60^{\circ} \mathrm{C}$. The obtained solid was filtered off and recrystallized from proper solvent.

(E)- $N$ '-(benzo[d][1,3] dioxol-5- ylmethylene)-2-(4-oxo-2- propylquinazolin-3 (4H) $-y l$ ) acetohydrazide (13a)

$\mathrm{IR}(\mathrm{KBr}) \mathrm{cm}^{-1}: 3200(\mathrm{NH}), 3055(\mathrm{CH}$ aromatic), 2962, 2931, 2870, $(\mathrm{CH}$ aliphatic), $1682\left(\mathrm{CH}_{2} \mathrm{C}=\mathrm{O}\right), 1675(\mathrm{C}=\mathrm{O})$ Quinazolinone), 1597, $1605(\mathrm{C}=\mathrm{N})$; MS: m/z 392 ,391, 377, 363, 349, 243, 308, 284, 270, 157, 143,144, 99. ; 1HNMR (DMSO-d6) : $\delta 9.21(\mathrm{~s}, \mathrm{H}, \mathrm{N}=\underline{\mathrm{CH}}), 7.21-8.12$ (m, 7H, aromatic), 6.33(s, 2H, $\left.\underline{\mathrm{CH}}_{2} \mathrm{CO}\right), 6.25$ (s, 2H, O- $\left.\mathrm{CH} 2-\mathrm{O}\right), 4.82$ (s, H, $\left.\underline{\mathrm{NH}}\right), 2.83\left(\mathrm{t}, 2 \mathrm{H}, \mathrm{CH}_{2} \mathrm{Q}\right)$ 1.52 (sextet, $\left.2 \mathrm{H}, \underline{\mathrm{CH}}_{2} \mathrm{Me}\right), 1.11\left(\mathrm{t}, 3 \mathrm{H}, \underline{\mathrm{CH}}_{3}\right)$.

(E)-N'-(4-hydroxybenzylidene)-2-(4-oxo-2-propylquinazolin-3(4H)-yl) acetohydrazide (13b)

IR(KBr) cm ${ }^{-1}: 4400(\mathrm{OH}), 3200(\mathrm{NH}), 3055$ (CH aromatic), 2962, 2931, 2870, ( $\mathrm{CH}$ aliphatic),1682 $\left(\mathrm{CH}_{2} \underline{\mathrm{C}=\mathrm{O}}\right), 1675(\mathrm{C}=\mathrm{O})$ Quinazolinone), 1597, $1605(\mathrm{C}=\mathrm{N})$; MS: m/z 365 [M+], 363, 347, 332, 314, 300, 243, 308, 284, 270, 157, 143,144, 99.

(E)-N'-(4-nitrobenzylidene)-2-(4-oxo-2-propylquinazolin-3(4H)-yl) acetohydrazide (13c)

$\mathrm{IR}(\mathrm{KBr}) \mathrm{cm}^{-1}: 3200(\mathrm{NH}), 3055(\mathrm{CH}$ aromatic $), 2962,2931,2870, \quad(\mathrm{CH}$ aliphatic), $1682\left(\mathrm{CH}_{2} \mathrm{C}=\mathrm{O}\right), 1675(\mathrm{C}=\mathrm{O})$ Quinazolinone $), 1597,1605(\mathrm{C}=\mathrm{N})$; MS: m/z 394 [M+] , 377, 363, 349, 308, 284, 270, 157, 143,144, 99 ; 1HNMR (DMSO-d6) : $\delta 9.21(\mathrm{~s}, \mathrm{H}, \mathrm{N}=\underline{\mathrm{CH}}), 7.21-8.12(\mathrm{~m}, 8 \mathrm{H}$, aromatic), 6.33 (s, 2H, $\left.\underline{\mathrm{CH}}_{2} \mathrm{CO}\right), 4.82$ (s, H, $\left.\underline{\mathrm{NH}}\right), 2.83\left(\mathrm{t}, 2 \mathrm{H}, \underline{\mathrm{CH}}_{2} \mathrm{Q}\right) 1.52$ (sextet, $2 \mathrm{H}, \underline{\mathrm{CH}}_{2} \mathrm{Me}$ ), 1.11(t, $\left.3 \mathrm{H}, \underline{\mathrm{CH}}_{3}\right)$.

$\mathrm{N}$-(4-nitro-1,3-dioxoisoindolin-yl)-2-(4-oxo-2-propylquinazolin-3(4H)-yl) acetamide (14)

A solution of hydrazide $9(0.01 \mathrm{~mol})$ and 3-nitrophthalic anhydride $(0.01 \mathrm{~mol})$ in ethanol was heated under reflux $(3 \mathrm{hr})$ the reaction mixture was allowed to cool and the obtained solid was filtered off and recrystallized from proper solvent. m.p. $250^{\circ} \mathrm{C} .3271(\mathrm{NH}), 3055$ (CH aromatic), 2962, 2931, 2870, $\quad(\mathrm{CH}$ Egypt. J. Chem. 53, No.6 (2010) 
aliphatic),1760, 1730, 1680, $1675(4 \mathrm{C}=\mathrm{O}), 1597,1605(\mathrm{C}=\mathrm{N}) ; \mathrm{MS}: \mathrm{m} / \mathrm{z} 436$ [M+],432, 317, 303, 189, 308, 284, 270, 157, 143, 144, 99.; 1HNMR (DMSOd6) : $\delta \quad 7.21-8.12\left(\mathrm{~m}, 7 \mathrm{H}\right.$, aromatic), $6.33\left(\mathrm{~s}, 2 \mathrm{H}, \underline{\mathrm{CH}_{2}} \mathrm{CO}\right), 4.82(\mathrm{~s}, \mathrm{H}, \underline{\mathrm{NH}})$, $2.83\left(\mathrm{t}, 2 \mathrm{H}, \underline{\mathrm{CH}_{2}} \mathrm{Q}\right) 1.52$ (sextet, $2 \mathrm{H}, \underline{\mathrm{CH}_{2}} \mathrm{Me}$ ) , 1.11(t, $\left.3 \mathrm{H}, \underline{\mathrm{CH}}_{3}\right)$.

\section{References}

1. Karel, W., Jii, G., Hynek, D., Jioi, K., Lenka, K., Vera, K. and Jarmila, K., Influence of the replacement of the oxo function with the thioxo group on the antimycobacterial activity of 3-aryl-6,8-dichloro-2H-1,3-benzoxazine-2,4(3H)-diones and 3-arylquinazoline -2,4(1H,3H)-diones). II Farmaco, 56, 803 (2001).

2. Sarvesh, K.P., Abhishek, S. and Ashutosh, S., Antimicrobial studies of some nove quinazolinones fused with [ 1,2,4 ]-triazole,[1,2,4]-triazine and [1,2,4,5]-tetrazine ring. Nizamuddin Eur. J. Med. Chem. 44, 1188 (2009)

3. EL-Tombary, A. A., Ismail, K. A., Aboulwafa, O. M., Omar, M. E., EL-Azzouni, M.Z. , EL-Mansoury, S.T. Novel triazolo [4,3-a]quinazolinone and bis-triazolo[4,3a : 4,3'-c]quinazolines : Synthesis and antitoxoplasmosis effect. II Farmaco, 54, 486 (1999).

4. Jen, T., Dienel, B., Dowalo, F., Hoeven, H.V., Bender, P. and Loev, B.، A midines. $5^{1}$. Synthesis of pyrrolo [2,3-b] isoquinoline imidazo[1,2-b]isoquinoline, pyrrolo[2,1-b]quinazoline and 1,3-thiazino [2,3-b]quinazoline derivatives and related heterocycles as potential antihypertensive agents. J. Med. Chem. 16, 633 (1973).

5. Alagarsamy, V., Solomon, V. R. and Murugan,M.، Amidines. $5^{1}$. Synthesis of pyrrolo [2,3-b] isoquinoline imidazo[1,2-b]isoquinoline, pyrrolo[2,1-b]quinazoline, and 1,3-thiazino [2,3-b]quinazoline derivatives and related heterocycles as potential antihypertensive agents. (Bio. Med. Chem. 15, 4009 (2007).

6. Alagarsamy, V., Giridhar, R. and Yadav, M.R.، Synthesis and pharmacological investigation of novel 1-substituted 4-(4-substitutid phenyl)-4H-[1,2.4]triazolo[4,3a]quinazolin-5-ones as a new class of $\mathrm{H}_{1}$-antihistaminc agents. J. Pharm. Pharmaco, 58, 1249 (2006).

7. Alagarsamy, V., Rupeshkumar,M., Kavitha,K., Meena,S., Shankar,D., Siddiqui, A. A. and Rajesh, R. Synthesis and pharmacological investigation of novel 4-(2methylphenyl)-1-substituted -4H-[1,2,4]triazolo[4,3-a]quinazolin-5-ones as new class of $\mathrm{H}_{1}$-antihistaminic agents. Eur. J. Med. Chem. 43, 2331(2008).

8. Alagarsamy, V., Shankar, D. and Murugesan, S., Synthesis of 4- butyl - 1substituted - $4 \mathrm{H}-[1,2,4]$ triazolo $\left[4,3\right.$-a] quinazolin-5-ones as new class of $\mathrm{H}_{1}$ antihistaminic agenta. Bio. Med. Pharm. 62, 173(2008) .

9. Alagarsamy, V., Giridhar, R. and Yadav, M.R.، Synthesis and pharmacological investigation of novel 1-substituted-4-phenyl-1, 2, 4-triazolo [4, 3-a] quinazolin$5(4 \mathrm{H})$-ones as a new class of $\mathrm{H}_{1}$-antihistaminic agents. Bio. Med. Chem. Lett. 15, 1877(2005) 
10. Varsha, J., Pradeep, M., Sushil, K. and Stables, J. P.، Synthesis and CNS depressant activity of some novel 3-[5-substituted 1, 3, 4-thiadiazole-2-yl]-2-styryl quinazoline-4(3H)ones. Eur. J. Med. Chem. 43, 135(2008).

11. Laddha, S. S. and Satyendra, P. B.، A new therapeutic approach in Parkinson's disease : some novel quinazoline derivatives as dual selective phospho diesterase 1 inhibitors and anti- inflammatory agents. Bio. Med. Chem. 17, 6796 (2009).

12. Guo, L., Kakarla, R., Samuel, W.G., Pendri, A. and Baoqing, M.` A facile one step synthesis of 5-chloro-imidazo $[1,5$-a]quinazoline by microwave irradiation. Tetrahedron Lett. 50, 6048 (2009).

13. Jen, H. M., Jiau, H. L. , Chu, K. S., Xia, Y., Bastow, K., Yuka, N., Hamel, N. Y. and Lee, K. H.، Alkylamino - and 2, 3-dihydro-3'-methoxy-2-phenyl-4quinazolinones and related compounds: Their synthesis, cytotoxicity, and inhibition of tubulin polymerization. J. Med. Chem. 43, 4479 (2000).

14. Laurent, F.A.H., Peter, B., Boyle, F.T., Delouvrie, B., Ellston, R.P.A., Halsall, C.T., Harris, C. S., Hudson, K., Kendrew, J., Pease, J. E., Ross, H. S., Smith, P. and Vincent, J. L., Novel 4-anilinoquinazolines with C-6 carbon-linked side chains : Synthesis and structure-activity relationship of a series of potent, orally active, EGF receptor tyrosine Kinase inhibitors. Bio. Med. Chem. Lett. 16, 2672 (2006).

15. Xia, Y., Yang, Z.Y., Hour, M.J., Kuo, S.C., Peng, X., Bastow, K. F., Nakanishi, Y., Priya, N., Hackl, T., Hamel, E. and Lee, K. H. , Antitumor agents . Part 204: Synthesis and biological evaluation of substituted 2-aryl quinazolinones. Bio. Med. Chem. Lett. 11 , 1193 (2001).

16. Kamel, A., Bharathi, E.V., Ramaiah, M. J., Dastagiri, D., Reddy, S., Viswanath, A., Sultana, F., Pushpavalli, S. N. C. V. L., Manika, P., Srivastava, H. K., Sastry, G. N. , Juvekar, A., Sen, S. and Zingde, S. ' Quinazolinone linked pyrrolo[2,1-c][1,4] benzodiazepine (PBD) conjugates : Design, synthesis and biological evaluation as potential anticancer agents. Bio. Med. Chem. 18, 526 (2010).

17. Zhou, Y., Murphy, D. E., Sun, Z. and Gregor, V. E., Novel parallel synthesis of N(4-oxo-2-substituted-4H-quinazolin-3-yl)-substituted sulfonamids. Tetrahedron Lett, 45, 8048 (2004).

18. Marsham, P.R., Hughes, L.R., Jackman, A.L., Hayter, A.J., Oldfield, J., Wardleworth, J. M., Bishop, J. A. M., O'Connor, B. M. and Calvert, A. H. Quinazoline antifolate thymidylate synthase inhibitors: Heterocyclic benzoyl ring modifications . J. Med. Chem. 34, 1594 (1991).

19. Alagarsamy, V., Solomon, V.R. and Dhanabal, K.، Synthesis and pharmacological evaluation of some3-phenyl -2-substituted-3H-quinazolin-4-one as analgesic, antiinflammatory agents. Bio. Med. Chem. 15, 235(2007).

20. Kumar, A., Sharma, S., Archana, Bajaj, K., Shipra, S., Panwar, H., Singh, T. and Srivastava, V. K. Some new 2,3,6-trisubstituted quinazolinones as potent antiinflammatory, analgesic and COX-II inhibitors. Bio. Med. Chem. 11, 5293 (2003) . 
21. Alagarsamy, V., Solomon, V.R., Murugan, M., Sankaranarayanan, R., Periyasamy, P., Deepa, R. and Anandkumar, T.D. Synthesis of 3-(2-Pyridyl)-2substituted-quinazolin-4(3H)-ones as new analgesic and anti-inflammatory agents. Bio. Med. Pharm. 62, 454 (2008).

22. Amin, K.M., Kamel, M.M., Anwar, M.M., Khedr, M. and Syam, Y.M.، Synthesis, biological evaluation and molecular docking of novel series of spiro $[(2 \mathrm{H}, 3 \mathrm{H})$ quinazoline-2,1'-cyclohexan $]-4(1 \mathrm{H})$-one derivatives as anti-inflammatory and analgesic agents . Eur. J. Med. Chem. xxx , 1(2010).

23. Archana , Srivastava, V. K. and Kumr, A. Synthesis of newer thiadiazolyl and thiazolidinonyl quinazolin $-4(3 \mathrm{H})$-ones as potential anticonvulsant agents. Eur. J. Med. Chem. 37 , 873(2002).

24. Archana, Srivastava, V. K. and Kumar, A., Synthesis of some newer derivatives of substituted quinazolinonyl-2-oxo $\backslash$ thiobarbituric acid as potent anticonvulsant agents. Bio. Med. Chem. 12, 1257(2004).

25. Kobayashi, S., Ueno, M., Suzuki, R. and Ishitani, H., Catalytic asymmetric synthesis of febrifugine and isofebrifugine . Tetrahedron Lett. 40, 2175 (1999).

26. Berman, E. M. and Werbel, L. M.، The renewed potential for folate antagonists in contemporary cancer chemotherapy. J. Med. Chem. 34, 479 (1991).

(Received $13 / 1 / 2010$;

accepted $20 / 6 / 2010)$ 


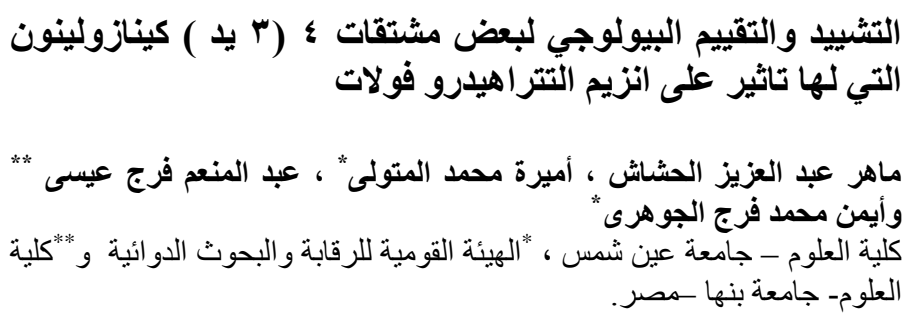

كل الكائنات الحية تحتاج الى انزيم التتراهيدروفولات اللازم لتخليق البيورين

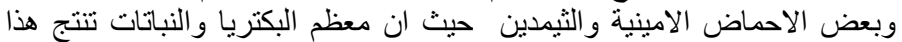

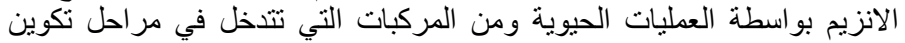
الانزيم ووجد انها تستخدم كمضادات للسرطان مركبر العبات الكبات الكينازولينون.

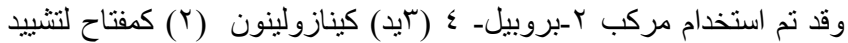

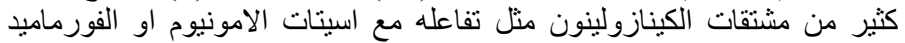

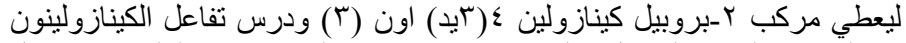

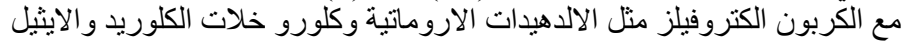

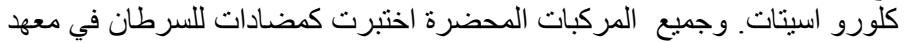
السرطان الامريكي ووجد ان بعض من هذه المركبات لها تاثير على الثى الخلايا

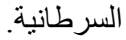

